\title{
New Handoff Control Method Using Fuzzy Multi-Criteria Decision Making in Micro/Pico-cellular Networks
}

\author{
Jong Chan Lee ${ }^{1}$, Dong Li ${ }^{1}$, Ki Hong Park ${ }^{1}$, and Hong-Jin Kim ${ }^{2}$ \\ ${ }^{1}$ Dept. of Computer Information Science, Kunsan National Univ., Korea \\ chan2000@kunsan.ac.kr \\ ${ }^{2}$ Dept. of Computer Information, KyungWon College, Korea \\ hjkim@kwc.ac.kr
}

\begin{abstract}
It is widely accepted that the coverage with high user densities can only be achieved with small cell such as Micro / Pico-cell. The smaller cell size causes frequent handoffs between cells and a decrease in the permissible handoff processing delay. This may result in the handoff failure, in addition to the loss of some packets during the handoff. In this paper we propose new handoff control scheme in the next generation mobile communications, in which the handoff setup process is done in advance before a handoff request by predicting the handoff cell based on mobile terminal's current position and moving direction.
\end{abstract}

\section{Introduction}

Next generation mobile communication systems are considered to support various types of high-speed multimedia traffic with packet switching at the same time. To do that, more upgraded quality of service and system capacity are needed. Due to the limitations of the radio spectrum, the next generation wireless networks will adopt Micro/Pico-cellular networks for various advantages including higher data throughput, greater frequency reuse, and location information with finer granularity. In this environment, because of small coverage area of Micro/Pico-cells, the handoff rate grows rapidly and fast handoff support is essential [1]. We propose a new method that makes it possible to avoid a handoff failure by performing the handoff setup process in advance before a handoff request in order to shorten the handoff delay, in which the handoff cell is selected based on the direction information from a block information database and the current position information from Position Estimator (PE) using Fuzzy Multi-Criteria Decision Making (FMCDM). To enhance estimation accuracy, we propose a scheme based on FMCDM which considers multiple parameters: the signal strength, the distance between the BS and a Mobile Terminal (MT), the moving direction, and the previous location. For predicting the MT's movement to the handover cell, we also propose the use of a block information database composed of block objects for mapping into the positional information provided by the PE. 


\section{Defining Location}

The position of a MT within a cell can be defined by dividing each cell into tracks and blocks, and relating these to the signal level received by it at that point. It is done automatically in two phases of track definition and block definition. Then the block information database is constructed with these results. The system scheme estimates in stepwise the optimal block at which the MT locates with the help of the block information database and the position information for PE. Three classified tracks are used to predict the mobility of the MT as shown in Fig. 1. Each cell consists of track_1 as a serving cell area, track_2 as a handoff cell selection area, and track_3 as a handover area, where the handoff area is defined to be the area where the received signal strength from the BS is between the handoff threshold and the acceptable received signal threshold. Within this area, a handoff is performed to the BS with the highest signal strength.

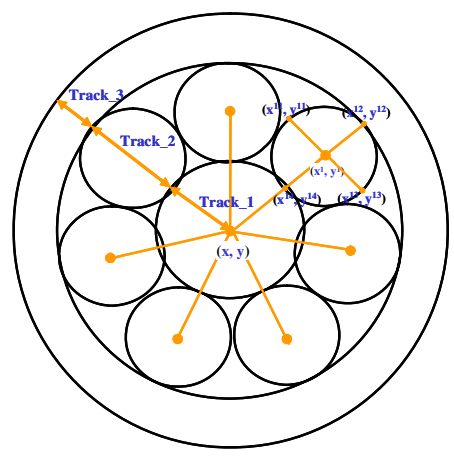

Fig. 1. Dividing a cell into tracks and Identifying the block using the vector

The collection of block information is called the block object. The block object contains the following information: BlockId, BlockLocationInfo indicating the information on the block's location within a cell comprised of one center point and four of area point; HandoffCellId indicating the adjacent cells to which a MT may hand over in this block; NextBlockId indicating another block within track_2 which may be traveled by a MT; VerificationRate indicating verification rate for the selected handover cells. Each MT updates periodically his mobile object which represents his current state for handover.

\section{Mobile Tracking Based on FMCDM}

In our method, the received signal strength $\left(P S S_{i}\right)$, the distance between the mobile and the base station $D_{i}$, the previous location $L_{i}$, and the moving direction $C_{i}$ are considered as decision parameters. Each decision problem involves $\mathrm{n}$ alternatives and $\mathrm{m}$ linguistic attributes corresponding to $\mathrm{m}$ criteria. Thus, decision data can be organized in $\mathrm{m}_{\mathrm{n}}$ matrix. The decision matrix for alternatives is given by Eq. (1). 


$$
\mu=\left[\begin{array}{cccc}
\mu_{R}\left(P S S_{11}\right) & \mu_{R}\left(D_{12}\right) & \mu_{R}\left(L_{13}\right) & \mu_{R}\left(C_{14}\right) \\
\mu_{R}\left(P S S_{21}\right) & \mu_{R}\left(D_{22}\right) & \mu_{R}\left(L_{23}\right) & \mu_{R}\left(C_{24}\right) \\
\mu_{R}\left(P S S_{31}\right) & \mu_{R}\left(D_{32}\right) & \mu_{R}\left(L_{33}\right) & \mu_{R}\left(C_{34}\right) \\
\ldots & \ldots & \ldots & \ldots \\
\mu_{R}\left(P S S_{n 1}\right) & \mu_{R}\left(D_{n 2}\right) & \mu_{R}\left(L_{n 3}\right) & \mu_{R}\left(C_{n 4}\right)
\end{array}\right]
$$

The weighting vector for evaluation criteria can be given by using linguistic terminology with fuzzy set theory [2-3]. Weighting vector $W$ is represented as Eq. (2).

$$
W=\left(w_{i}^{P S S}, w_{i}^{D}, w_{i}^{L}, w_{i}^{C}\right)
$$

By multiplying the weighting vector by the decision matrix, the performance matrix is given by Eq. (3).

GMV (Generalized Mean Value) [2-3] is used for ranking the alternatives according to the aggregated criterion. The GMV for alternatives is represented as Eq. (4).

$$
m\left(\mu_{n}\right)=\frac{\left(C_{i}+D_{i}\right)^{2}-\left(A_{i}+B_{i}\right)^{2}+A_{i} \cdot B_{i}-C_{i} \cdot D_{i}}{3 \cdot\left[\left(C_{i}+D_{i}\right)-\left(A_{i}+B_{i}\right)\right]}
$$

where $A_{i}=\mu_{R}\left(P S S_{n 1}\right) \times w_{i}^{P S S}, B_{i}=\mu_{R}\left(D_{n 2}\right) \times w_{i}^{D}$, and $C_{i}=\mu_{R}\left(L_{n 3}\right) \times w_{i}^{L}, D_{i}=\mu_{R}$ $\left(C_{n m}\right) \times w_{i}^{C}$ respectively.

\section{Direction Based Handoff Method}

\subsection{Selection of Handoff Cells}

The basic principle of the handoff cell selection is list as follow:

1. Position information creation and the measurement of the received signal stren gths from the active cell and Surrounding cells, the PE determines MT's positio $\mathrm{n}$ based FMCDM. First candidate cell set is obtained from the measurement of $\mathrm{t}$ he downlink channel quality of the active cell and the surrounding cells.

2. Block selection, system selects the corresponding block object by comparing th e computed position by PE with the BlockLocationInfo of each block object. Ha ndoff cell set is obtained from the selected block object.

3. The first effectiveness inspection, the first effectiveness inspection between the $\mathrm{f}$ irst candidate cell set and the handover cell set is done. The exception handling is done if there is no correspondence between the two.

4. Handoff cell selection, if an effectiveness inspection for the selected block is co mpleted, handoff cells are determined from HandoffCell Id information of the $\mathrm{b}$ lock object.

5. The registration of the handoff cell information, the handoff cell information is registered to a cell management table. 


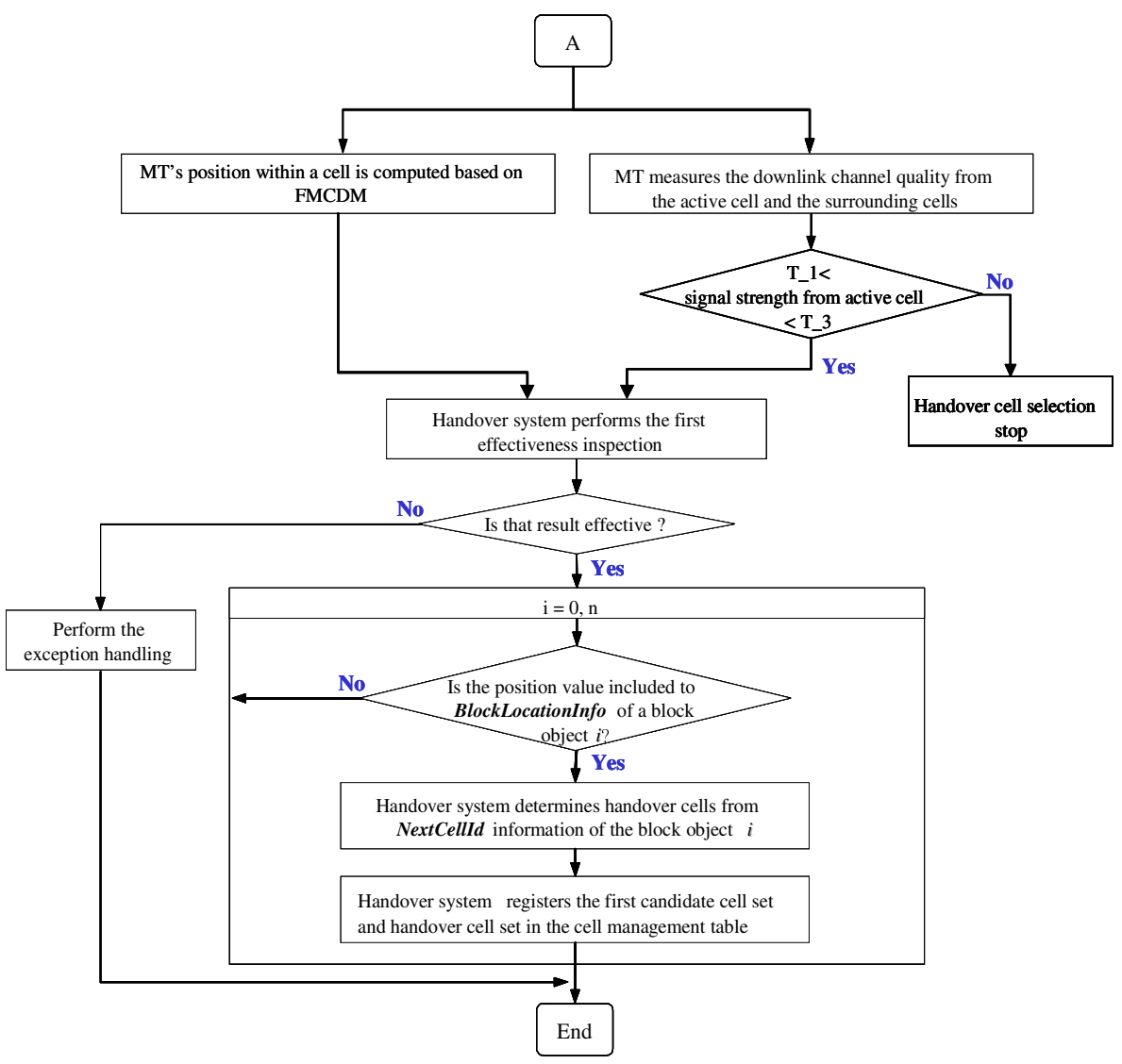

Fig. 2. Flowchart of a handoff cell selection algorithm

Fig. 2 shows a handoff cell selection procedure. Each MT's position information from PE is valid only in track_2. Therefore, the handoff cell selection process is terminated if a MT is located at other tracks. The first effectiveness inspection between the first candidate cell set and the handoff cell set is done, and if there is no correspondence between the two, the exception handling is performed. If one more cells are same, Handoff system selects an optimum handoff cell based on the resource availability.

\subsection{Handoff Pre-processing}

Using information on the MT's handover cells determined from the above handoff cell selection algorithm, two level handoff process, radio level and network level, is performed as shown in Fig. 3.

- The radio level handoff process is performed for the conversion of radio link modem reconfiguration, synchronization setting and so forth - from previous access point to new access point.

- The network level handoff process is performed for packet buffering and rerouting, for the purpose of supporting the radio level handoff. 


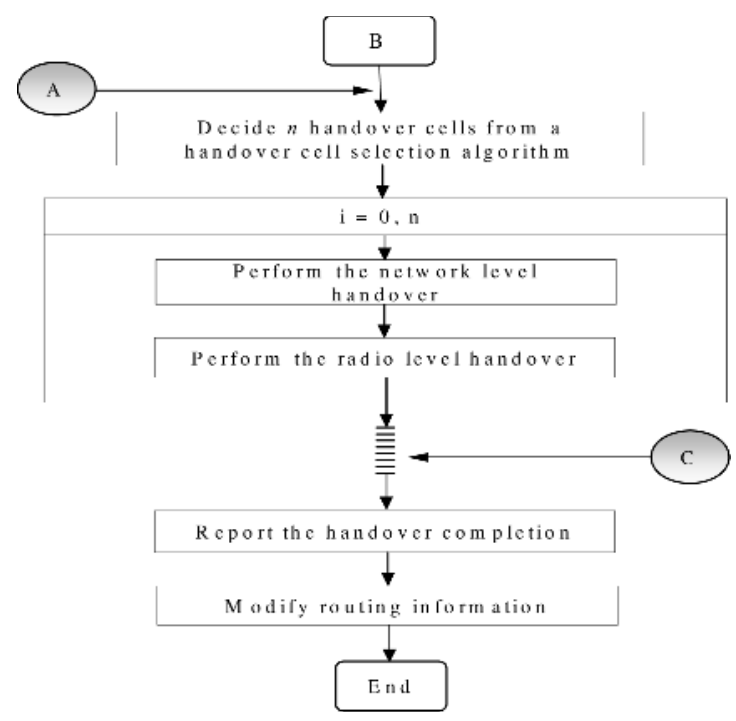

Fig. 3. The flowchart of a handoff pre-processing procedure

\subsection{Handoff Decision}

For a handoff decision, a MT will search the neighboring BSs using the information on the handoff cells selected from the above handoff cell selection algorithm. Three types of handoff can be provided, namely forward handoff, backward handoff and reconfiguration. A forward handoff is done if the handoff cell set is identical with the set of the second candidate cells, and backward handoff with MAHO procedure is done if the handoff cell set does not correspond with the second candidate cell set, and reconfiguration is done if the position traveled by the MT is another block within track_2. The MT reports his handoff completion to a handover system through the target cell, and the handoff system requests the release of the connections related with the MT. An old cell releases all the resource allocated for the MT, reports the result to the handoff system.

\section{Simulation Results}

Fig. 4 (a) shows the handoff failure rate versus the session arrival rate. The solid curve represents the prediction based handoff method applied and the dashed curve represents a previous handoff method. The major cause of the handoff failure is because of the prediction error caused by PE. It can be seen that the prediction error does very largely with increase in the PE error. The proposed method performs the handoff setup process in advance before a handoff request by predicting the handoff cell based on each MT's current position so that the handoff failure can be reduced. Fig. 4 (b) shows the effect of the proposed method on packet loss rate. It is observed that the proposed method provides a noticeable improvement over the conventional 


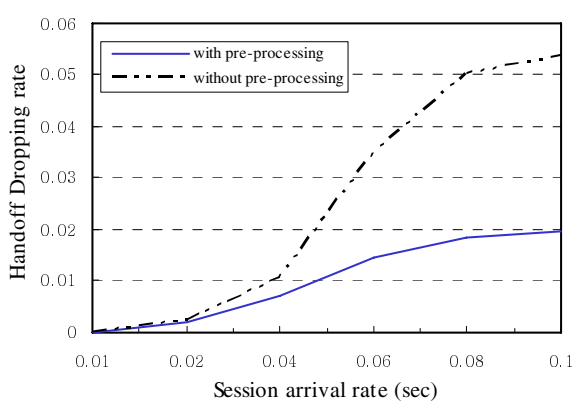

(a) Handoff dropping rate

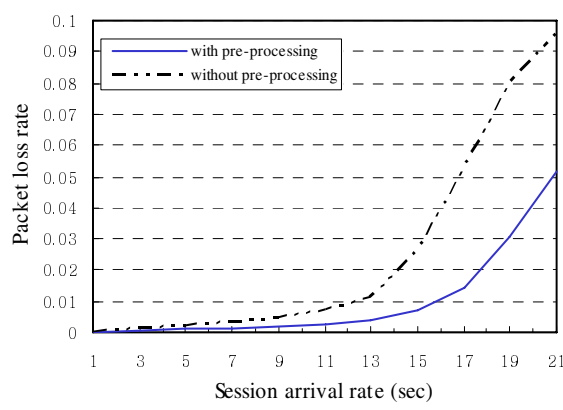

(b) Packet loss rate

Fig. 4. The comparison of handoff failure rate and packet loss rate

scheme, because the MT has already established synchronization to the BS in target cell and switches its Tx to target BS while stop communicating with the original BS at the same time after the cell search procedure so that there will be data lost for uplink of 2 4 frames due to the uplink synchronization and there's no data lost for downlink.

\section{Conclusion}

This paper main goal is to address the problem of handoff failure for MTs as they move from one position to another at high speeds in small cell environment. In this scheme, the handoff connection setup process is established prior to the handoff request. The handoff cell is predicted by the MT's position and direction and a database that includes the MT's position information. Further researches are required on their implementation and applications to the handoff.

\section{References}

1. M. Ergen, S. Coleri, B. Dundar, A. Puri, J. Walrand, and P. Varaiya, "Position Leverage Smooth Handover Algorithm", IEEE ICN 2002, Atlanta, 2002.

2. C. Naso and B. Turchiano, "A Fuzzy Multi-Criteria Algorithm for Dynamic Routing in FMS," IEEE ICSMC'1998, Vol. 1, pp. 457-462, 1998.

3. C. H. Yeh and H. Deng, "An Algorithm for Fuzzy Multi-Criteria Decision Making," IEEE ICIPS'1997, pp. 1564-1568, 1997. 